\title{
How patients understand depression associated with chronic physical disease - a systematic review
}

\author{
Sarah L Alderson*, Robbie Foy, Liz Glidewell, Kate McLintock and Allan House
}

\begin{abstract}
Background: Clinicians are encouraged to screen people with chronic physical illness for depression. Screening alone may not improve outcomes, especially if the process is incompatible with patient beliefs. The aim of this research is to understand people's beliefs about depression, particularly in the presence of chronic physical disease.

Methods: A mixed method systematic review involving a thematic analysis of qualitative studies and quantitative studies of beliefs held by people with current depressive symptoms.

MEDLINE, EMBASE, PSYCHINFO, CINAHL, BIOSIS, Web of Science, The Cochrane Library, UKCRN portfolio,

National Research Register Archive, Clinicaltrials.gov and OpenSIGLE were searched from database inception to $31^{\text {st }}$ December 2010.

A narrative synthesis of qualitative and quantitative data, based initially upon illness representations and extended to include other themes not compatible with that framework.

Results: A range of clinically relevant beliefs was identified from 65 studies including the difficulty in labeling depression, complex causal factors instead of the biological model, the roles of different treatments and negative views about the consequences of depression. We found other important themes less related to ideas about illness: the existence of a self-sustaining 'depression spiral'; depression as an existential state; the ambiguous status of suicidal thinking; and the role of stigma and blame in depression.
\end{abstract}

Conclusions: Approaches to detection of depression in physical illness need to be receptive to the range of beliefs held by patients. Patient beliefs have implications for engagement with depression screening.

Keywords: Depression, Comprehension, Primary health care, Chronic disease, Review, Systematic

\section{Background}

Clinicians are often encouraged to identify emotional problems in patients with physical disorders. For example, guidance from the UK National Institute for Health and Clinical Excellence (NICE) states that "screening should be undertaken in primary care ...for depression in highrisk groups" [1]. Up to a quarter of people with diabetes or coronary heart disease have depression $[2,3]$ which is associated with poorer outcomes [4]. Policy initiatives in the UK have included financial incentives for general practitioners to screen all patients with coronary heart disease or diabetes [5] and expanded access to psychological services for people with long-term physical

\footnotetext{
* Correspondence: s.l.alderson@leeds.ac.uk

Institute of Health Sciences, University of Leeds, Leeds, UK
}

conditions [6]. Over $92 \%$ of eligible patients were screened in 2009-10 [7]. However, it is uncertain whether incentivising screening alone has improved patient outcomes [8].

Three conditions need to be satisfied for screening to improve outcomes: engagement of primary care staff with the screening process [9]; a systematic approach to patient management following detection [10-12], and patient engagement with the screening process. This review concentrates on the third condition which is relatively neglected in the previously published literature. Consultation models emphasize the importance of understanding patient perspectives in clinical care [13]. This may be particularly challenging in states such as depression which lack clear cut diagnostic boundaries, and in the context of depression screening in physical illness which usually takes place at chronic disease clinics, or

\section{() BioMed Central}


opportunistically during other consultations, where time to explore patient beliefs is often limited. Beliefs will also influence the subsequent management of depression including adherence to anti-depressant medication regimes and referral to specialist mental health services [14-16].

We conducted a mixed methods systematic review of studies of people with current depressive symptoms, which investigated their beliefs about those symptoms.

\section{Methods}

\section{Search strategy}

The review protocol can be requested from the study authors. We sought English-language studies of adults with current depressive symptoms that reported beliefs about depression. We systematically searched for articles and included studies of beliefs associated with chronic physical illness in stage 1 and then systematically searched for studies that included all depression beliefs in stage 2. Searches were limited to primary care where that was possible using the database search terms. We excluded non-English language studies and studies that assessed subjects without current depression or, explored beliefs about other mental health disorders (including anxiety, post-natal depression or bipolar disorder). Figure 1 detail our search methods [17]. Appendix 1 details the search terms applied. We (SA) also reviewed reference lists of all included studies.

Initial screening of titles and abstracts, with exclusion of those that were obviously not related to depression beliefs, was undertaken by one reviewer (SA) with $18 \%$ (913) of studies reviewed by a second reviewer (KM).
Full-text articles were assessed in detail by two reviewers (SA and KM) for all potentially eligible studies. All disagreements between reviewers were resolved by discussion.

\section{Data extraction and quality assessment}

Data on study participants, methods and findings were abstracted from included studies using a standardised form specifically developed for this review. The findings of qualitative studies were entered verbatim into NVivo8, a qualitative data analysis software package. We assessed study quality using established criteria [18]. Authors were contacted for further information as required.

\section{Data synthesis}

We conducted a narrative synthesis [19]. This approach to the synthesis of evidence relies primarily on the use of words and text to summarise and explain the findings of multiple studies. It is especially suited to a study like ours in which there is wide variation in study type included. Stage one involved a thematic and content analysis of the qualitative data. We initially categorised beliefs about depression using Leventhal's Illness Representations [20], a framework for characterising patients' beliefs about illnesses [21-23].

The illness representation includes five main categories of belief: identity (beliefs concerning label and associated symptoms), cause (factors and conditions believed to have caused a condition), timeline (acute, cyclical or chronic), consequences (expected effects on physical, social and psychological well-being) and the control and/or cure (to what extent treatment and behaviours will help), along

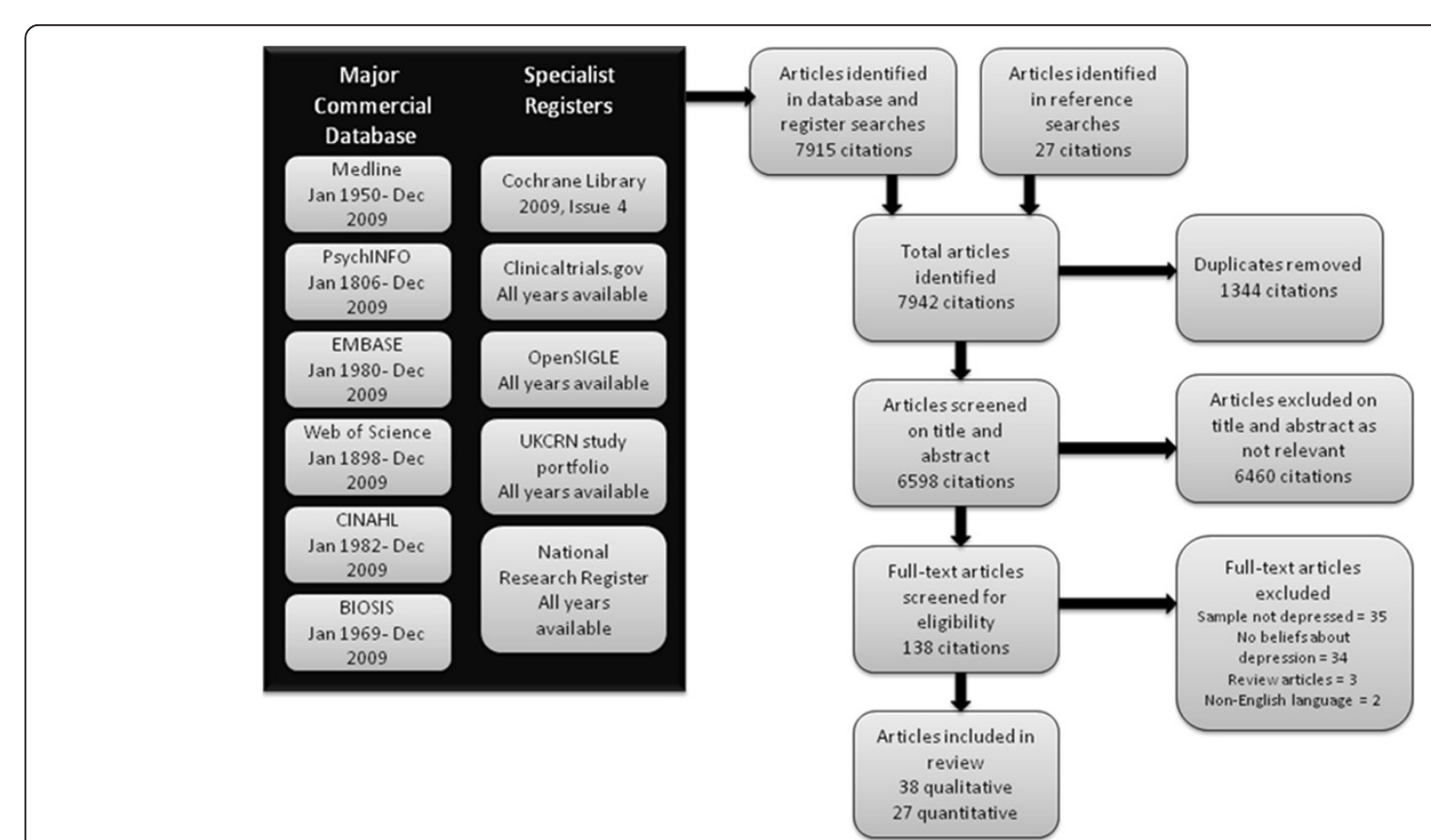

Figure 1 PRISMA chart of search strategy and identification of publications included in the review. 
with a parallel emotional representation. We also identified beliefs not adequately captured by the framework and developed new themes which were agreed by consensus. The coding of themes was checked for $10 \%$ of studies by a second researcher.

Reviews of the quantitative findings were mapped onto the framework derived from the qualitative literature. For example, the finding that $68 \%$ of participants in one study felt that having depression changed the way they viewed themselves [24] supported the theme of existential \& self.

In stage two we assessed the robustness of the synthesis by appraising the contribution of weak studies to review findings. Quality was assessed using criteria appropriate to study design [18]. Studies were assigned a score and topics based upon weak studies only were not included in the final analysis.

The final stage involved integrating the findings from the preceding stages into overarching conclusions.

\section{Ethical approval}

This project did not require ethical approval.

\section{Results}

We identified 7942 abstracts, of which 64 individual studies from 65 reports were included (Figure 1). Table 1 summarizes all included studies. Studies ranged widely in terms of questions addressed and methods used with 37 studies using qualitative interviews and 27 using self-administered questionnaires. The majority of the studies took place in the UK or the United States. Less than half (45\%) applied theoretical frameworks to collect or analyze data, with Leventhal's Illness Representations being the most commonly used in both qualitative and quantitative studies. Beliefs about depression associated with chronic physical diseases were identified specifically in only two qualitative studies; however participants in other studies referred to physical ill health in their beliefs. We therefore addressed all beliefs about depression within a single synthetic review. No studies were excluded from the review because of poor quality.

Beliefs could be coded to all the main categories of illness representation. We developed five new thematic categories for beliefs that did not fit well into the illness representations framework. We labeled these: understandability; the depression cycle, existential and self, suicidal thinking and stigma, blame and responsibility. Table 2 shows the studies that contributed to each theme. Figure 2 shows themes with their associated subthemes.

\section{Identity}

Depression, depressed and depressive were the most commonly used labels by both authors and study participants. Other labels used by participants were stress, blues, nerves, sadness, loneliness and emotional or mental disorder.

Some participants said they would rather not know they had depression, whereas others believed the label meant treatment was possible and they were not 'mad'. Some felt depression or its symptoms were a normal part of life and not a disease and that the word 'depression' is used in everyday language without meaning an illness.

"It never occurred to me that I could be depressed,

I just thought that I was a nasty person." [64]

"Did I know what it was? It was pain, but I don't

think I would have called it depression. I think I would have called it my pain." [60]

\section{Cause}

Most study participants could name at least one cause for their depression and many had complex, multifactorial causes. Most participants believed the causes were external and took the form of significant negative life events and stress rather than subscribing to a mainly biological model. Co-existing physical illnesses were mentioned by several participants. Where the biological model was mentioned it was either to disagree with it or to mention it alongside other causal beliefs.

"I have diabetes and other people with diabetes have experience with depression because of our treatment, and the things we have to do, and the way we have to live now [which is] different from the way we were used to doing things before." [59]

"I think it [depression] is due to a lack of hormones, that is, a consequence of being exposed to stress over such a long period of time." [44]

\section{Cure and/or control}

Study participants found it difficult to acknowledge the need for help; however, many believed the GP was the right person to approach. Participants had strong beliefs over whether medication or talking therapies would help them. Some mentioned beliefs about alternative therapies such as St. John's wort or using prayer, often used alone if the depression was not seen as having a severe impact upon their life. A few participants were unable to identify any cure or control and some felt that depression is incurable.

"When I talk about my problems I think about them and I feel worse. So I don't know if it really is better to talk about them because you remember all your problems. Sometimes I feel better when I am doing other things and not talking about it to anyone." [58] "Well I think it's a waste of time really, he'll just give me yet another pill and I shall still be depressed because of all the other things that are wrong with me." [43] 
Table 1 Table of included Studies

\begin{tabular}{|c|c|c|c|c|c|c|c|c|c|}
\hline $\begin{array}{l}\text { Study \& Year } \\
\text { of Publication }\end{array}$ & Country & Gender & Ethnicity & Setting & $\begin{array}{l}\text { Research } \\
\text { Approach }\end{array}$ & $\begin{array}{l}\text { Theoretical } \\
\text { Framework }\end{array}$ & $\begin{array}{l}\text { Depression } \\
\text { status }\end{array}$ & $\begin{array}{l}\text { Quality } \\
\text { Assessment }\end{array}$ & Aim \\
\hline ADDIS 1995 [25] & USA & Female $>$ Male & not given & not given & $\begin{array}{l}\text { Cross-sectional } \\
\text { Survey }\end{array}$ & $\begin{array}{l}\text { Reasons for } \\
\text { Depression }\end{array}$ & $\begin{array}{l}\text { Diagnostic } \\
\text { Interview }\end{array}$ & $C$ & $\begin{array}{l}\text { To develop the Reasons For } \\
\text { Depression questionnaire and } \\
\text { measure its internal consistency } \\
\text { and validity }\end{array}$ \\
\hline ADDIS 1996 [26] & USA & not given & not given & $\begin{array}{l}\text { Primary Care } \\
\& \text { Community }\end{array}$ & $\begin{array}{l}\text { Cross-sectional } \\
\text { Survey }\end{array}$ & None & $\begin{array}{l}\text { Diagnostic } \\
\text { Interview }\end{array}$ & C & $\begin{array}{l}\text { To examine the relationships } \\
\text { between clients reasons for } \\
\text { depression and the outcome } \\
\text { of treatment }\end{array}$ \\
\hline ALLEN 1998 [27] & UK & Male $=$ Female & not given & Secondary Care & $\begin{array}{l}\text { Cross-sectional } \\
\text { Survey }\end{array}$ & None & $\begin{array}{l}\text { Screening } \\
\text { Test }\end{array}$ & B & $\begin{array}{l}\text { To examine the presence of } \\
\text { depressive symptoms as well } \\
\text { as attitudes to and knowledge } \\
\text { of depression in a group of } \\
\text { physically ill inpatients }\end{array}$ \\
\hline AL-SAFFAR 2003 [28] & Kuwait & Male $>$ Female & Arabic & Secondary Care & $\begin{array}{l}\text { Cross-sectional } \\
\text { Survey }\end{array}$ & $\begin{array}{l}\text { Health Belief } \\
\text { Model }\end{array}$ & $\begin{array}{l}\text { Diagnostic } \\
\text { Interview }\end{array}$ & B & $\begin{array}{l}\text { To determine whether underlying } \\
\text { attitudes and health beliefs of } \\
\text { patients were affecting their } \\
\text { decision to take their medication } \\
\text { as prescribed }\end{array}$ \\
\hline $\begin{array}{l}\text { BACKENSTRASS } \\
2007[29]\end{array}$ & Germany & Female $>$ Male & not given & Primary Care & $\begin{array}{l}\text { Semi-structured } \\
\text { Interview }\end{array}$ & none & $\begin{array}{l}\text { Diagnostic } \\
\text { Interview }\end{array}$ & B & $\begin{array}{l}\text { GP and sub-threshold depression } \\
\text { patients views on diagnosis } \\
\text { \& treatment }\end{array}$ \\
\hline BADGER 2007b [30] & UK & Female $>$ Male & not given & Primary Care & $\begin{array}{l}\text { Semi-structured } \\
\text { Interview }\end{array}$ & none & $\begin{array}{l}\text { Medical } \\
\text { Records }\end{array}$ & B & $\begin{array}{l}\text { Attitudes towards and use of } \\
\text { self-chosen treatment in patients } \\
\text { prescribed antidepressants }\end{array}$ \\
\hline BANN 2004 [31] & USA & Female $>$ Male & Mixed & Secondary Care & $\begin{array}{l}\text { Cross-sectional } \\
\text { Survey }\end{array}$ & $\begin{array}{l}\text { Explanatory } \\
\text { Model }\end{array}$ & $\begin{array}{l}\text { Screening } \\
\text { Test }\end{array}$ & C & $\begin{array}{l}\text { To evaluate the psychometric } \\
\text { properties of the EMD instrument }\end{array}$ \\
\hline BOGNER 2008 [32] & USA & Female $>$ Male & Mixed & Primary Care & $\begin{array}{l}\text { Semi-structured } \\
\text { Interview }\end{array}$ & none & $\begin{array}{l}\text { Screening } \\
\text { Test }\end{array}$ & A & $\begin{array}{l}\text { Older patients' perspectives on } \\
\text { the relationship of heart disease } \\
\text { to depression }\end{array}$ \\
\hline BROWN 2001 [24] & USA & Female $>$ Male & Mixed & Primary Care & $\begin{array}{l}\text { Cross-sectional } \\
\text { Survey }\end{array}$ & CS-SRM IR & $\begin{array}{l}\text { Screening } \\
\text { Test }\end{array}$ & C & $\begin{array}{l}\text { To determine whether primary } \\
\text { care patients' personal illness } \\
\text { cognitions for depression are } \\
\text { associated with depression coping } \\
\text { strategies and treatment related } \\
\text { behavior }\end{array}$ \\
\hline BROWN 2005 [33] & USA & Female $>$ Male & White & Primary Care & $\begin{array}{l}\text { Cross-sectional } \\
\text { Survey }\end{array}$ & CS-SRM IR & $\begin{array}{l}\text { Medical } \\
\text { Records }\end{array}$ & B & $\begin{array}{l}\text { To describe beliefs about } \\
\text { antidepressants, examine the } \\
\text { factor structure of the BMQ }\end{array}$ \\
\hline BROWN 2007 [34] & USA & Female $>$ Male & White & Primary Care & $\begin{array}{l}\text { Cross-sectional } \\
\text { Survey }\end{array}$ & CS-SRM IR & $\begin{array}{l}\text { Medical } \\
\text { Records }\end{array}$ & B & $\begin{array}{l}\text { to describe personal illness models } \\
\text { for depression and the relationship } \\
\text { with functional disability }\end{array}$ \\
\hline
\end{tabular}


Table 1 Table of included Studies (Continued)

\begin{tabular}{|c|c|c|c|c|c|c|c|c|c|}
\hline $\begin{array}{l}\text { BURROUGHS } \\
2006 \text { [35] }\end{array}$ & UK & Both & not given & Primary Care & $\begin{array}{l}\text { Semi-structured } \\
\text { Interview }\end{array}$ & none & $\begin{array}{l}\text { Screening } \\
\text { Test }\end{array}$ & B & $\begin{array}{l}\text { Primary care professionals ideas } \\
\text { about depression in elderly and } \\
\text { elderly views on depression as a } \\
\text { problem \& help-seeking }\end{array}$ \\
\hline CABASSA 2008 [36] & USA & Female $>$ Male & Hispanic & Primary Care & $\begin{array}{l}\text { Cross-sectional } \\
\text { Survey }\end{array}$ & CS-SRM IR & $\begin{array}{l}\text { Screening } \\
\text { Test }\end{array}$ & C & $\begin{array}{l}\text { The aim of the present study is to } \\
\text { conduct a confirmatory factor } \\
\text { analysis (CFA) of the IPQR adapted } \\
\text { for a clinical sample of depressed } \\
\text { low-income Latinos served in } \\
\text { primary care. }\end{array}$ \\
\hline CAPE 1999 [37] & UK & Female $>$ Male & not given & Primary Care & $\begin{array}{l}\text { Semi-structured } \\
\text { Interview }\end{array}$ & none & $\begin{array}{l}\text { Screening } \\
\text { Test }\end{array}$ & C & $\begin{array}{l}\text { Patients' reasons for not discussing } \\
\text { emotional problems with GP }\end{array}$ \\
\hline $\begin{array}{l}\text { CHAKRABORTY } \\
2009[38]\end{array}$ & India & Male $=$ Female & Indian & Secondary Care & $\begin{array}{l}\text { Cross-sectional } \\
\text { Survey }\end{array}$ & None & $\begin{array}{l}\text { Diagnostic } \\
\text { Interview }\end{array}$ & B & $\begin{array}{l}\text { Attitudes and beliefs of patients of } \\
\text { first episode depression towards } \\
\text { antidepressant treatment and the } \\
\text { relationship between beliefs and } \\
\text { treatment adherence }\end{array}$ \\
\hline COOPER 1998 [39] & USA & Female $>$ Male & Mixed & Primary Care & $\begin{array}{l}\text { Cross-sectional } \\
\text { Survey }\end{array}$ & None & $\begin{array}{l}\text { Diagnostic } \\
\text { Interview }\end{array}$ & B & $\begin{array}{l}\text { To compare the views of African- } \\
\text { American and white adult primary } \\
\text { care patients regarding the } \\
\text { importance of various aspects of } \\
\text { depression care }\end{array}$ \\
\hline COOPER 2000 [40] & USA & Female $>$ Male & Mixed & Primary Care & $\begin{array}{l}\text { Cross-sectional } \\
\text { Survey }\end{array}$ & None & $\begin{array}{l}\text { Screening } \\
\text { Test }\end{array}$ & B & $\begin{array}{l}\text { To select items for inclusion in an } \\
\text { instrument to measure attitudes } \\
\text { towards depression care }\end{array}$ \\
\hline COOPER 2003 [41] & USA & Female $>$ Male & Mixed & Primary Care & $\begin{array}{l}\text { Cross-sectional } \\
\text { Survey }\end{array}$ & $\begin{array}{l}\text { Theory of } \\
\text { Reasoned Action }\end{array}$ & $\begin{array}{l}\text { Diagnostic } \\
\text { Interview }\end{array}$ & B & $\begin{array}{l}\text { The objective of this study was to } \\
\text { examine whether racial and ethnic } \\
\text { differences exist in patient } \\
\text { attitudes toward depression care. }\end{array}$ \\
\hline $\begin{array}{l}\text { COOPER-PATRICK } \\
1997 \text { [42] }\end{array}$ & USA & Female $>$ Male & Mixed & Primary Care & Focus Group & none & $\begin{array}{l}\text { Medical } \\
\text { Records }\end{array}$ & B & $\begin{array}{l}\text { Health professionals and patients' } \\
\text { identifying attitudes that influence } \\
\text { help-seeking }\end{array}$ \\
\hline CORNFORD 2007 [43] & UK & Female $>$ Male & not given & Primary Care & $\begin{array}{l}\text { Semi-structured } \\
\text { Interview }\end{array}$ & none & $\begin{array}{l}\text { Screening } \\
\text { Test }\end{array}$ & A & $\begin{array}{l}\text { Lay beliefs about depression } \\
\text { symptoms and how they manage } \\
\text { them }\end{array}$ \\
\hline $\begin{array}{l}\text { DANIELSSON } \\
2009[44]\end{array}$ & Sweden & Equal & Swedish & Primary Care & $\begin{array}{l}\text { Semi-structured } \\
\text { Interview }\end{array}$ & Gender Theory & $\begin{array}{l}\text { Medical } \\
\text { Records }\end{array}$ & A & $\begin{array}{l}\text { To explore how primary care } \\
\text { patients experience \& understand } \\
\text { depression and the impact of } \\
\text { gender in this process }\end{array}$ \\
\hline DEJMAN 2008 [45] & Iran & Female & Middle-Eastern & Secondary Care & $\begin{array}{l}\text { Semi-structured } \\
\text { Interview }\end{array}$ & $\begin{array}{l}\text { Explanatory } \\
\text { Model }\end{array}$ & $\begin{array}{l}\text { Medical } \\
\text { Records \& } \\
\text { Screening } \\
\text { Test }\end{array}$ & B & $\begin{array}{l}\text { Explanatory models of help-seeking } \\
\text { and coping with depression in } \\
\text { Iranian women }\end{array}$ \\
\hline EDLUND 2008 [46] & USA & Male $>$ Female & Mixed & Primary Care & $\begin{array}{l}\text { Randomised } \\
\text { Controlled Trial }\end{array}$ & $\begin{array}{l}\text { Health Belief } \\
\text { Model }\end{array}$ & $\begin{array}{l}\text { Screening } \\
\text { Test }\end{array}$ & B & $\begin{array}{l}\text { To assess the extent to which } \\
\text { beliefs changed in the intervention } \\
\text { and treatment as usual arms of } \\
\text { the study }\end{array}$ \\
\hline
\end{tabular}


Table 1 Table of included Studies (Continued)

\begin{tabular}{|c|c|c|c|c|c|c|c|c|c|}
\hline FORTUNE 2004 [47] & UK & Female & not given & not given & $\begin{array}{l}\text { Qualitative Writing } \\
\& \text { Cross-sectional } \\
\text { survey }\end{array}$ & CS-SRM IR & $\begin{array}{l}\text { Screening } \\
\text { Test }\end{array}$ & C & $\begin{array}{l}\text { To compare the structure and } \\
\text { content of peoples models of } \\
\text { depression with those of a physical } \\
\text { illness }\end{array}$ \\
\hline GARFIELD 2003 [48] & UK & Female $>$ Male & Mixed & Primary Care & $\begin{array}{l}\text { Semi-structured } \\
\text { Interview }\end{array}$ & none & $\begin{array}{l}\text { Medical } \\
\text { Records }\end{array}$ & A & $\begin{array}{l}\text { To identify factors of importance } \\
\text { to patients when beginning } \\
\text { courses of antidepressant } \\
\text { treatment }\end{array}$ \\
\hline GASK 2003 [49] & UK & Female $>$ Male & not given & Primary Care & $\begin{array}{l}\text { Semi-structured } \\
\text { Interview }\end{array}$ & none & $\begin{array}{l}\text { Medical } \\
\text { Records }\end{array}$ & B & $\begin{array}{l}\text { To explore depressed patients' } \\
\text { perceptions of the quality of care } \\
\text { from GP's }\end{array}$ \\
\hline GIVENS 2006 [50] & USA & Female $>$ Male & not given & Primary Care & $\begin{array}{l}\text { Semi-structured } \\
\text { Interview }\end{array}$ & $\begin{array}{l}\text { Explanatory } \\
\text { Model }\end{array}$ & $\begin{array}{l}\text { Diagnostic } \\
\text { Interview }\end{array}$ & B & $\begin{array}{l}\text { To understand why older people } \\
\text { are adverse to using antidepressants }\end{array}$ \\
\hline GIVENS 2007 [51] & USA & Female $>$ Male & Mixed & Online & $\begin{array}{l}\text { Cross-sectional } \\
\text { Survey }\end{array}$ & None & $\begin{array}{l}\text { Screening } \\
\text { Test }\end{array}$ & B & $\begin{array}{l}\text { To describe ethnic differences in } \\
\text { attitudes toward depression }\end{array}$ \\
\hline GREEN 2002 [52] & UK & Female & Chinese & $\begin{array}{l}\text { Primary \& } \\
\text { Secondary Care } \\
\& \text { Community }\end{array}$ & $\begin{array}{l}\text { Semi-structured } \\
\text { Interview }\end{array}$ & none & $\begin{array}{l}\text { Screening } \\
\text { Test }\end{array}$ & B & $\begin{array}{l}\text { To identify barriers to Chinese } \\
\text { women accessing help for } \\
\text { depression }\end{array}$ \\
\hline GRIME 2003 [53] & UK & Female $>$ Male & not given & $\begin{array}{l}\text { Primary Care } \\
\& \text { Community }\end{array}$ & $\begin{array}{l}\text { Semi-structured } \\
\text { Interview }\end{array}$ & none & $\begin{array}{l}\text { Medical } \\
\text { Records or } \\
\text { Self Diagnosis }\end{array}$ & B & $\begin{array}{l}\text { To understand patients views } \\
\text { and experiences of taking } \\
\text { antidepressants }\end{array}$ \\
\hline HEIFNER 1997 [54] & USA & Male & not given & not given & $\begin{array}{l}\text { Semi-structured } \\
\text { Interview }\end{array}$ & none & $\begin{array}{l}\text { Medical } \\
\text { Records }\end{array}$ & B & $\begin{array}{l}\text { To explore the male experience of } \\
\text { depression }\end{array}$ \\
\hline KANGAS 2001 [55] & Finland & Female $>$ Male & not given & Community & $\begin{array}{l}\text { Semi-structured } \\
\text { Interview }\end{array}$ & $\begin{array}{l}\text { Narrative } \\
\text { Reconstruction }\end{array}$ & Self Diagnosis & C & $\begin{array}{l}\text { To discover how people explain the } \\
\text { cause of their depression }\end{array}$ \\
\hline KARASZ 2003 [56] & USA & Female $>$ Male & Mixed & Primary Care & $\begin{array}{l}\text { Semi-structured } \\
\text { Interview }\end{array}$ & CS-SRM IR & $\begin{array}{l}\text { Screening } \\
\text { Test }\end{array}$ & B & $\begin{array}{l}\text { To explore patients' conceptual } \\
\text { labels of depression and build } \\
\text { a theoretical } \\
\text { model linking these to attitudes to } \\
\text { treatment }\end{array}$ \\
\hline KARASZ 2006 [57] & USA & Female $>$ Male & Hispanic & Primary Care & $\begin{array}{l}\text { Semi-structured } \\
\text { Interview }\end{array}$ & none & $\begin{array}{l}\text { Screening } \\
\text { Test }\end{array}$ & C & $\begin{array}{l}\text { To investigate Hispanic patients' } \\
\text { perceptions of primary care } \\
\text { treatments for depression }\end{array}$ \\
\hline KARASZ 2008 [58] & USA & Female > Male & Hispanic & Primary Care & $\begin{array}{l}\text { Semi-structured } \\
\text { Interview }\end{array}$ & CS-SRM IR & $\begin{array}{l}\text { Screening } \\
\text { Test }\end{array}$ & B & $\begin{array}{l}\text { To explore the heterogeneity of } \\
\text { depression experience }\end{array}$ \\
\hline KARASZ 2009 [59] & USA & Female $>$ Male & not given & Primary Care & $\begin{array}{l}\text { Semi-structured } \\
\text { Interview }\end{array}$ & CS-SRM IR & $\begin{array}{l}\text { Screening } \\
\text { Test }\end{array}$ & A & $\begin{array}{l}\text { To examine conceptual models of } \\
\text { depression in different ethnic } \\
\text { groups and focusing on the } \\
\text { degree to which patients } \\
\text { conceptual models matched } \\
\text { a bio-psychiatric model }\end{array}$ \\
\hline KARP 1994 [60] & UK & Female $>$ Male & White British & Secondary Care & $\begin{array}{l}\text { Semi-structured } \\
\text { Interview }\end{array}$ & Illness Career & $\begin{array}{l}\text { Medical } \\
\text { Records }\end{array}$ & A & $\begin{array}{l}\text { How those suffering from uni-polar } \\
\text { depression perceive, interpret, } \\
\text { and understand a life condition } \\
\text { that often seems incoherent, } \\
\text { fragmented and intractable }\end{array}$ \\
\hline
\end{tabular}


Table 1 Table of included Studies (Continued)

\begin{tabular}{|c|c|c|c|c|c|c|c|c|c|}
\hline KELLY 2007 [61] & USA & Female $>$ Male & not given & Primary Care & $\begin{array}{l}\text { Cross-sectional } \\
\text { Survey }\end{array}$ & CS-SRM IR & $\begin{array}{l}\text { Medical } \\
\text { Records }\end{array}$ & B & $\begin{array}{l}\text { To examine the relationships } \\
\text { between beliefs about depression } \\
\text { and emotion }\end{array}$ \\
\hline KIRK 2001 [62] & USA & Female & Mixed & Primary Care & $\begin{array}{l}\text { Cross-sectional } \\
\text { Survey }\end{array}$ & None & $\begin{array}{l}\text { Diagnostic } \\
\text { Interview }\end{array}$ & $C$ & $\begin{array}{l}\text { Assessed pre-existing attitudes to } \\
\text { depression and its treatment in a } \\
\text { population of economically } \\
\text { disadvantaged women }\end{array}$ \\
\hline KUYKEN 1992 [63] & UK & Female $>$ Male & not given & Secondary Care & $\begin{array}{l}\text { Semi-structured } \\
\text { Interview \& } \\
\text { Cross-sectional } \\
\text { Survey }\end{array}$ & none & $\begin{array}{l}\text { Screening } \\
\text { Test }\end{array}$ & C & $\begin{array}{l}\text { To investigate beliefs and attitudes } \\
\text { towards depression in patients' and } \\
\text { compare them to lay people and } \\
\text { psychologists }\end{array}$ \\
\hline LEWIS 1995 [64] & UK & not given & not given & $\begin{array}{l}\text { Primary \& } \\
\text { Secondary Care } \\
\text { \& Community }\end{array}$ & $\begin{array}{l}\text { Semi-structured } \\
\text { Interview }\end{array}$ & none & $\begin{array}{l}\text { Medical } \\
\text { Records or } \\
\text { Self Diagnosis }\end{array}$ & B & $\begin{array}{l}\text { To investigate the experience of } \\
\text { depression as a meaningful } \\
\text { experience }\end{array}$ \\
\hline LEYKIN 2007 [65] & USA & not given & not given & Secondary Care & $\begin{array}{l}\text { Randomised } \\
\text { Controlled Trial }\end{array}$ & $\begin{array}{l}\text { Reasons for } \\
\text { Depression }\end{array}$ & $\begin{array}{l}\text { Diagnostic } \\
\text { Interview }\end{array}$ & $C$ & $\begin{array}{l}\text { To look at relation between beliefs } \\
\text { and outcomes of therapies }\end{array}$ \\
\hline LOWE 2006 [66] & Germany & Female $>$ Male & not given & Secondary Care & $\begin{array}{l}\text { Semi-structured } \\
\text { Interview }\end{array}$ & none & $\begin{array}{l}\text { Diagnostic } \\
\text { Interview }\end{array}$ & C & $\begin{array}{l}\text { To investigate attitudes towards } \\
\text { treatment approaches }\end{array}$ \\
\hline MANBER 2003 [67] & USA & Female $>$ Male & Mixed & Secondary Care & $\begin{array}{l}\text { Cross-sectional } \\
\text { Survey }\end{array}$ & CS-SRM IR & $\begin{array}{l}\text { Diagnostic } \\
\text { Interview }\end{array}$ & C & $\begin{array}{l}\text { To develop the Perception of } \\
\text { Depressive illness questionnaire }\end{array}$ \\
\hline MARTIN 2007a [67] & Brazil & Female & Brazilian & Secondary Care & $\begin{array}{l}\text { Ethnographic } \\
\text { observation \& } \\
\text { Semi-structured } \\
\text { Interview }\end{array}$ & none & $\begin{array}{l}\text { Medical } \\
\text { Records }\end{array}$ & B & $\begin{array}{l}\text { To describe the perception of } \\
\text { depression for women in Embu, } \\
\text { Sao Paulo }\end{array}$ \\
\hline MARTIN 2007b [68] & Brazil & Female & Brazilian & Secondary Care & $\begin{array}{l}\text { Ethnographic } \\
\text { observation \& } \\
\text { Semi-structured } \\
\text { Interview }\end{array}$ & none & $\begin{array}{l}\text { Medical } \\
\text { Records }\end{array}$ & B & $\begin{array}{l}\text { To assess the meaning of } \\
\text { depression in women diagnosed } \\
\text { with the disorder, and the context } \\
\text { of care given by the psychiatrists }\end{array}$ \\
\hline MAXWELL 2005 [69] & UK & Female & not given & Primary Care & $\begin{array}{l}\text { Semi-structured } \\
\text { Interview }\end{array}$ & none & $\begin{array}{l}\text { Medical } \\
\text { Records }\end{array}$ & A & $\begin{array}{l}\text { To explore GP and patients' } \\
\text { accounts of recognizing and } \\
\text { treating depression }\end{array}$ \\
\hline NOLAN 2005 [70] & UK & Female $>$ Male & not given & Primary Care & $\begin{array}{l}\text { Semi-structured } \\
\text { Interview }\end{array}$ & none & $\begin{array}{l}\text { Medical } \\
\text { Records }\end{array}$ & B & $\begin{array}{l}\text { To identify how patients treated } \\
\text { with medication for their } \\
\text { depression perceived the } \\
\text { relationship with their prescribing } \\
\text { clinician }\end{array}$ \\
\hline OKELLO 2007 [71] & Uganda & Female $>$ Male & African & Secondary Care & $\begin{array}{l}\text { Semi-structured } \\
\text { Interview }\end{array}$ & $\begin{array}{l}\text { Explanatory } \\
\text { Model }\end{array}$ & $\begin{array}{l}\text { Medical } \\
\text { Records }\end{array}$ & A & $\begin{array}{l}\text { To examine depressed patients' } \\
\text { perception of depression }\end{array}$ \\
\hline PANG 1998 [72] & USA & Female $>$ Male & Korean & Community & $\begin{array}{l}\text { Semi-structured } \\
\text { Interview }\end{array}$ & none & $\begin{array}{l}\text { Diagnostic } \\
\text { Interview }\end{array}$ & C & $\begin{array}{l}\text { To explore the ways depression } \\
\text { symptoms are expressed by elderly } \\
\text { Korean women }\end{array}$ \\
\hline
\end{tabular}


Table 1 Table of included Studies (Continued)

\begin{tabular}{|c|c|c|c|c|c|c|c|c|c|}
\hline ROGERS 2001 [73] & UK & Female $>$ Male & not given & Primary Care & $\begin{array}{l}\text { Semi-structured } \\
\text { Interview }\end{array}$ & none & $\begin{array}{l}\text { Medical } \\
\text { Records }\end{array}$ & B & $\begin{array}{l}\text { to explore experiences of depressed } \\
\text { people with their contact with } \\
\text { primary care }\end{array}$ \\
\hline SARKISIAN 2003 [74] & USA & Male $=$ Female & Mixed & Primary Care & $\begin{array}{l}\text { Cross-sectional } \\
\text { Survey }\end{array}$ & None & $\begin{array}{l}\text { Screening } \\
\text { Test }\end{array}$ & B & $\begin{array}{l}\text { To determine whether older adults } \\
\text { who attribute their depression to } \\
\text { aging are less likely to believe } \\
\text { seeking help is important }\end{array}$ \\
\hline $\begin{array}{l}\text { SCATTOLON } \\
1999[75]\end{array}$ & Canada & Female & not given & Community & $\begin{array}{l}\text { Semi-structured } \\
\text { Interview }\end{array}$ & none & $\begin{array}{l}\text { Self } \\
\text { Diagnosis }\end{array}$ & A & $\begin{array}{l}\text { Explore experiences of depression } \\
\text { and their ways of coping }\end{array}$ \\
\hline SHIN 2002 [76] & USA & Female $>$ Male & Korean & Community & $\begin{array}{l}\text { Semi-structured } \\
\text { Interview \& } \\
\text { Focus Groups }\end{array}$ & none & $\begin{array}{l}\text { Self } \\
\text { Diagnosis }\end{array}$ & A & $\begin{array}{l}\text { To investigate Korean Immigrants' } \\
\text { help-seeking behaviours for } \\
\text { depression \& under-utilization of } \\
\text { mental health services }\end{array}$ \\
\hline SRINIVASAN 2003 [77] & Canada & Female $>$ Male & not given & Secondary Care & $\begin{array}{l}\text { Cross-sectional } \\
\text { Survey }\end{array}$ & None & $\begin{array}{l}\text { Medical } \\
\text { Records }\end{array}$ & B & $\begin{array}{l}\text { The implications for patients } \\
\text { perspectives for treatment } \\
\text { preference, delivery \& medication } \\
\text { compliance }\end{array}$ \\
\hline STECKER 2007 [78] & USA & Female $>$ Male & Mixed & Primary Care & $\begin{array}{l}\text { Cross-sectional } \\
\text { Survey }\end{array}$ & None & $\begin{array}{l}\text { Medical } \\
\text { Records }\end{array}$ & B & $\begin{array}{l}\text { To investigate whether attitudes } \\
\text { towards psychotherapy in a } \\
\text { population of primary care patients } \\
\text { diagnosed with depression } \\
\text { influenced the likelihood that they } \\
\text { initiated psychotherapy }\end{array}$ \\
\hline UGARRIZA 2002 [79] & USA & Female & not given & Secondary Care & $\begin{array}{l}\text { Semi-structured } \\
\text { Interview }\end{array}$ & $\begin{array}{l}\text { Explanatory } \\
\text { Model }\end{array}$ & $\begin{array}{l}\text { Medical } \\
\text { Records }\end{array}$ & B & $\begin{array}{l}\text { What is the explanation of } \\
\text { depression given by a group of } \\
\text { older women with depression }\end{array}$ \\
\hline $\begin{array}{l}\text { VAN VOORHEES } \\
2005 \text { [80] }\end{array}$ & USA & Female $>$ Male & Mixed & Online & $\begin{array}{l}\text { Cross-sectional } \\
\text { Survey }\end{array}$ & $\begin{array}{l}\text { Theory of } \\
\text { Reasoned Action }\end{array}$ & $\begin{array}{l}\text { Screening } \\
\text { Test }\end{array}$ & B & $\begin{array}{l}\text { To develop a multivariate model of } \\
\text { intent not to accept a diagnosis } \\
\text { of depression }\end{array}$ \\
\hline $\begin{array}{l}\text { VAN VOORHEES } \\
2006 \text { [81] }\end{array}$ & USA & Female $>$ Male & Mixed & Online & $\begin{array}{l}\text { Cross-sectional } \\
\text { Survey }\end{array}$ & $\begin{array}{l}\text { Theory of } \\
\text { Reasoned Action }\end{array}$ & $\begin{array}{l}\text { Screening } \\
\text { Test }\end{array}$ & B & $\begin{array}{l}\text { Specifically, we examine the } \\
\text { relationship between five types of } \\
\text { actors and low self-perceived need } \\
\text { for treatment: beliefs and attitudes } \\
\text { towards treatment behaviors, } \\
\text { subjective social norms, past } \\
\text { treatment behaviors, illness factors, } \\
\text { and personal characteristics. }\end{array}$ \\
\hline WAGNER 1999 [82] & USA & Female $>$ Male & Mixed & Community & $\begin{array}{l}\text { Semi-structured } \\
\text { Interview }\end{array}$ & none & $\begin{array}{l}\text { Self } \\
\text { Diagnosis }\end{array}$ & B & $\begin{array}{l}\text { To examine the reasons people } \\
\text { choose to self-medicate with } \\
\text { St. John's wort instead of seeking } \\
\text { care from a conventional health } \\
\text { care provider. }\end{array}$ \\
\hline
\end{tabular}


Table 1 Table of included Studies (Continued)

\begin{tabular}{|c|c|c|c|c|c|c|c|c|c|}
\hline WAITE 2009 [83] & USA & Female & African American & Primary Care & Focus Group & $\begin{array}{l}\text { Explanatory } \\
\text { Model }\end{array}$ & $\begin{array}{l}\text { Medical } \\
\text { Records }\end{array}$ & A & $\begin{array}{l}\text { To examine the explanatory models } \\
\text { for depression among a cohort } \\
\text { of low-income African American } \\
\text { women }\end{array}$ \\
\hline WILLIAMS 2001 [84] & UK & not given & not given & Primary Care & $\begin{array}{l}\text { Semi-structured } \\
\text { Interview }\end{array}$ & CS-SRM IR & $\begin{array}{l}\text { Medical } \\
\text { Records }\end{array}$ & A & $\begin{array}{l}\text { To explore the perceptions of cause } \\
\text { of psychological distress }\end{array}$ \\
\hline WITTINK 2008 [85] & USA & Female $>$ Male & Mixed & Primary Care & $\begin{array}{l}\text { Semi-structured } \\
\text { Interview }\end{array}$ & $\begin{array}{l}\text { Cultural Models } \\
\text { Theory }\end{array}$ & $\begin{array}{l}\text { Medical } \\
\text { Records }\end{array}$ & A & $\begin{array}{l}\text { To identify health beliefs about } \\
\text { depression in older adults and how } \\
\text { they perceive differ from doctors }\end{array}$ \\
\hline WITTKAMPF 2008 [86] & Netherlands & Equal & Mixed & Primary Care & $\begin{array}{l}\text { Semi-structured } \\
\text { Interview }\end{array}$ & none & $\begin{array}{l}\text { Diagnostic } \\
\text { Interview }\end{array}$ & B & $\begin{array}{l}\text { To understand the views of patients' } \\
\text { who screened positive in a } \\
\text { depression screening programme }\end{array}$ \\
\hline YEUNG 2004 [87] & USA & Female $>$ Male & Chinese & Primary Care & $\begin{array}{l}\text { Cross-sectional } \\
\text { Survey }\end{array}$ & $\begin{array}{l}\text { Explanatory } \\
\text { Model }\end{array}$ & $\begin{array}{l}\text { Diagnostic } \\
\text { Interview }\end{array}$ & C & $\begin{array}{l}\text { To use the Explanatory Model } \\
\text { Interview Catalogue to examine } \\
\text { systematically the illness beliefs of } \\
\text { depressed Chinese American } \\
\text { patients seeking treatment at a } \\
\text { primary care clinic. }\end{array}$ \\
\hline
\end{tabular}


Table 2 Table of themes identified in each included study

\begin{tabular}{|c|c|c|c|c|c|c|c|c|c|}
\hline Study & Identity & Cause & $\begin{array}{l}\text { Cure/ } \\
\text { Control }\end{array}$ & Consequences & Timeline & Understandability & $\begin{array}{l}\text { Depression } \\
\text { Cycle }\end{array}$ & $\begin{array}{l}\text { Existential Suicide } \\
\& \text { Self }\end{array}$ & $\begin{array}{l}\text { Stigma, blame } \\
\text { \& responsibility }\end{array}$ \\
\hline ADDIS 1195 & & $\checkmark$ & & & & & & & \\
\hline ADDIS 1196 & & $\checkmark$ & $\checkmark$ & & & & & & \\
\hline ALLEN 1998 & $\checkmark$ & & $\checkmark$ & & & & & & \\
\hline AL-SAFFAR 2003 & & $\checkmark$ & $\checkmark$ & $\checkmark$ & & & & & \\
\hline BACKENSTRASS 2007 & $\checkmark$ & $\checkmark$ & $\checkmark$ & & $\checkmark$ & & & & $\checkmark$ \\
\hline BADGER 2007b & $\checkmark$ & $\checkmark$ & $\checkmark$ & & $\checkmark$ & & & & \\
\hline BANN 2004 & & $\checkmark$ & $\checkmark$ & & $\checkmark$ & & & & \\
\hline BOGNER 2008 & $\checkmark$ & $\checkmark$ & $\checkmark$ & $\checkmark$ & & $\checkmark$ & & & \\
\hline BROWN 2001 & $\checkmark$ & $\checkmark$ & $\checkmark$ & $\checkmark$ & & & & $\checkmark$ & $\checkmark$ \\
\hline BROWN 2005 & & & $\checkmark$ & & & & & & \\
\hline BROWN 2007 & $\checkmark$ & $\checkmark$ & $\checkmark$ & $\checkmark$ & $\checkmark$ & & & $\checkmark$ & $\checkmark$ \\
\hline BURROUGHS 2006 & $\checkmark$ & $\checkmark$ & $\checkmark$ & & $\checkmark$ & $\checkmark$ & & & $\checkmark$ \\
\hline CABASSA 2008 & $\checkmark$ & $\checkmark$ & $\checkmark$ & $\checkmark$ & $\checkmark$ & & & & \\
\hline CAPE 1999 & $\checkmark$ & $\checkmark$ & $\checkmark$ & & $\checkmark$ & & & & $\checkmark$ \\
\hline CHAKRABORTY 2009 & & $\checkmark$ & $\checkmark$ & & & & & & \\
\hline COOPER 1998 & $\checkmark$ & & $\checkmark$ & & & & & & $\checkmark$ \\
\hline COOPER 2000 & $\checkmark$ & & $\checkmark$ & & & & & & $\checkmark$ \\
\hline COOPER 2003 & & & $\checkmark$ & & & & & & $\checkmark$ \\
\hline COOPER-PATRICK 1997 & $\checkmark$ & $\checkmark$ & $\checkmark$ & $\checkmark$ & & & & & $\checkmark$ \\
\hline CORNFORD 2007 & $\checkmark$ & $\checkmark$ & $\checkmark$ & $\checkmark$ & $\checkmark$ & & $\checkmark$ & $\checkmark$ & $\checkmark$ \\
\hline DANIELSSON 2009 & $\checkmark$ & $\checkmark$ & $\checkmark$ & $\checkmark$ & $\checkmark$ & $\checkmark$ & & $\checkmark$ & $\checkmark$ \\
\hline DEJMAN 2008 & $\checkmark$ & & $\checkmark$ & & & & & & \\
\hline EDLUND 2008 & $\checkmark$ & & $\checkmark$ & & & & & & $\checkmark$ \\
\hline FORTUNE 2004 & $\checkmark$ & $\checkmark$ & $\checkmark$ & $\checkmark$ & $\checkmark$ & & & & \\
\hline GARFIELD 2003 & $\checkmark$ & $\checkmark$ & $\checkmark$ & $\checkmark$ & & & & & $\checkmark$ \\
\hline GASK 2003 & $\checkmark$ & & $\checkmark$ & & & & & & $\checkmark$ \\
\hline GIVENS 2006 & $\checkmark$ & $\checkmark$ & $\checkmark$ & & $\checkmark$ & & & & $\checkmark$ \\
\hline GIVENS 2007 & $\checkmark$ & $\checkmark$ & $\checkmark$ & $\checkmark$ & & & & & $\checkmark$ \\
\hline GREEN 2002 & $\checkmark$ & $\checkmark$ & $\checkmark$ & & & & & & $\checkmark$ \\
\hline GRIME 2003 & $\checkmark$ & $\checkmark$ & $\checkmark$ & & $\checkmark$ & & & & \\
\hline HEIFNER 1997 & $\checkmark$ & $\checkmark$ & $\checkmark$ & $\checkmark$ & $\checkmark$ & & & & $\checkmark$ \\
\hline KANGAS 2001 & $\checkmark$ & $\checkmark$ & $\checkmark$ & $\checkmark$ & $\checkmark$ & $\checkmark$ & $\checkmark$ & & $\checkmark$ \\
\hline KARASZ 2003 & $\checkmark$ & $\checkmark$ & $\checkmark$ & $\checkmark$ & $\checkmark$ & & & & \\
\hline KARASZ 2006 & $\checkmark$ & $\checkmark$ & $\checkmark$ & $\checkmark$ & $\checkmark$ & & & & \\
\hline KARASZ 2008 & $\checkmark$ & $\checkmark$ & $\checkmark$ & $\checkmark$ & $\checkmark$ & & & & \\
\hline KARASZ 2009 & $\checkmark$ & $\checkmark$ & $\checkmark$ & $\checkmark$ & $\checkmark$ & & $\checkmark$ & $\checkmark$ & \\
\hline KARP 1994 & $\checkmark$ & $\checkmark$ & $\checkmark$ & $\checkmark$ & $\checkmark$ & & & $\checkmark$ & $\checkmark$ \\
\hline KELLY 2007 & $\checkmark$ & $\checkmark$ & $\checkmark$ & $\checkmark$ & $\checkmark$ & & & & \\
\hline KIRK 2001 & $\checkmark$ & & $\checkmark$ & $\checkmark$ & $\checkmark$ & & & & $\checkmark$ \\
\hline KUYKEN 1992 & $\checkmark$ & $\checkmark$ & $\checkmark$ & & & & & & \\
\hline LEWIS 1995 & $\checkmark$ & & $\checkmark$ & & $\checkmark$ & & & & $\checkmark$ \\
\hline LEYKIN 2007 & & $\checkmark$ & & & & & & & \\
\hline LOWE 2006 & $\checkmark$ & $\checkmark$ & $\checkmark$ & & & & & & \\
\hline MANBER 2003 & $\checkmark$ & $\checkmark$ & $\checkmark$ & $\checkmark$ & $\checkmark$ & & & & \\
\hline MARTIN 2007a & $\checkmark$ & $\checkmark$ & $\checkmark$ & $\checkmark$ & $\checkmark$ & & & & $\checkmark$ \\
\hline MARTIN 2007b & $\checkmark$ & $\checkmark$ & $\checkmark$ & $\checkmark$ & $\checkmark$ & & & & \\
\hline
\end{tabular}


Table 2 Table of themes identified in each included study (Continued)

\begin{tabular}{|c|c|c|c|c|c|c|c|}
\hline MAXWELL 2005 & $\checkmark$ & & $\checkmark$ & $\checkmark$ & $\checkmark$ & & $\checkmark$ \\
\hline NOLAN 2005 & $\checkmark$ & & $\checkmark$ & $\checkmark$ & $\checkmark$ & & $\checkmark$ \\
\hline OKELLO 2007 & $\checkmark$ & $\checkmark$ & $\checkmark$ & $\checkmark$ & $\checkmark$ & & $\checkmark$ \\
\hline PANG 1998 & $\checkmark$ & $\checkmark$ & $\checkmark$ & $\checkmark$ & $\checkmark$ & & $\checkmark$ \\
\hline ROGERS 2001 & $\checkmark$ & $\checkmark$ & $\checkmark$ & $\checkmark$ & $\checkmark$ & $\checkmark$ & $\checkmark$ \\
\hline SARKISIAN 2003 & & $\checkmark$ & $\checkmark$ & & & & \\
\hline SCATOLLON 1999 & $\checkmark$ & $\checkmark$ & $\checkmark$ & $\checkmark$ & $\checkmark$ & & $\checkmark$ \\
\hline SHIN 2002 & $\checkmark$ & $\checkmark$ & $\checkmark$ & $\checkmark$ & $\checkmark$ & & $\checkmark$ \\
\hline SRINIVASAN 2003 & & $\checkmark$ & & & & & \\
\hline STECKER 2007 & & & $\checkmark$ & & & & \\
\hline UGARRIZA 2002 & $\checkmark$ & $\checkmark$ & $\checkmark$ & $\checkmark$ & $\checkmark$ & & \\
\hline VAN VOORHEES 2005 & $\checkmark$ & $\checkmark$ & $\checkmark$ & $\checkmark$ & & & $\checkmark$ \\
\hline VAN VOORHEES 2006 & & $\checkmark$ & $\checkmark$ & & & & $\checkmark$ \\
\hline WAGNER 1999 & $\checkmark$ & $\checkmark$ & $\checkmark$ & & $\checkmark$ & & \\
\hline WAITE 2009 & $\checkmark$ & $\checkmark$ & $\checkmark$ & $\checkmark$ & & $\checkmark$ & $\checkmark$ \\
\hline WILLIAMS 2001 & $\checkmark$ & $\checkmark$ & $\checkmark$ & & $\checkmark$ & & \\
\hline WITTINK 2008 & $\checkmark$ & $\checkmark$ & $\checkmark$ & & & & \\
\hline WITTKAMPF 2008 & $\checkmark$ & $\checkmark$ & $\checkmark$ & $\checkmark$ & $\checkmark$ & $\checkmark$ & $\checkmark$ \\
\hline YEUNG 2004 & $\checkmark$ & $\checkmark$ & $\checkmark$ & & & & $\checkmark$ \\
\hline
\end{tabular}

\section{Timeline}

The timescales participants mentioned for onset, duration and response to treatment were reported as varying markedly with acute, cyclical and chronic timescales being mentioned. The onset was described as "a bolt out of the blue" or "slow and insidious". Treatment response was seen as a short or long process.

"I am so afraid that I am going to remain depressed.

That is the only thing I fear." [79]

"Well, if it's only something that's going to be short term, then obviously it's worth getting the help and then sort your problems out and see how things go after that, more or less." [69]

\section{Consequences}

Depression was seen as having mostly negative consequences, affecting all present and future aspects of life, including work, social and home life and physical health, especially where there was a co-existing illness. Some held particular fears of losing control and embarrassing themselves.

"So you'll be dying of sadness, you get that sadness because the doctors say that if you stay really sad you begin to get other types of diseases like those that come from anguish, sadness, from depression you go on getting other types of disease and you end up dying too. Besides depression, it sets off other systems within your organism and ends up killing you." [88]
"I was already on a pedestal, being the first doctor ever in the family, and my mom and dad didn't want this to take me down from that pedestal in the other family's eyes." [54]

\section{Emotions}

We could not distinguish an emotional representation for depression from emotional symptoms of depression. Participants associated depression emotionally with fear, anger, sadness, despair, and guilt.

"Anxiety, anger, confusion, frustration for me is associated with the depression. Not sadness so much." [59]

"I'm afraid. . of being an invalid. . not doing the things I want to do." [32]

\section{Understandability}

Participants' beliefs about depression were not always internally consistent. Some understood their depression in terms of their life story and gave coherent beliefs. Thereby, a woman who believed the cause of her depression was her poor eyesight that stopped her from doing things believed the cure for this was to "get her eyes sorted" [35]. For others depression was "unexplained" and "not understood" which led to conflicting and less fixed beliefs. For example, one study reported of people with depression "Their explanations changed within their narratives and they tried out several explanations, not 


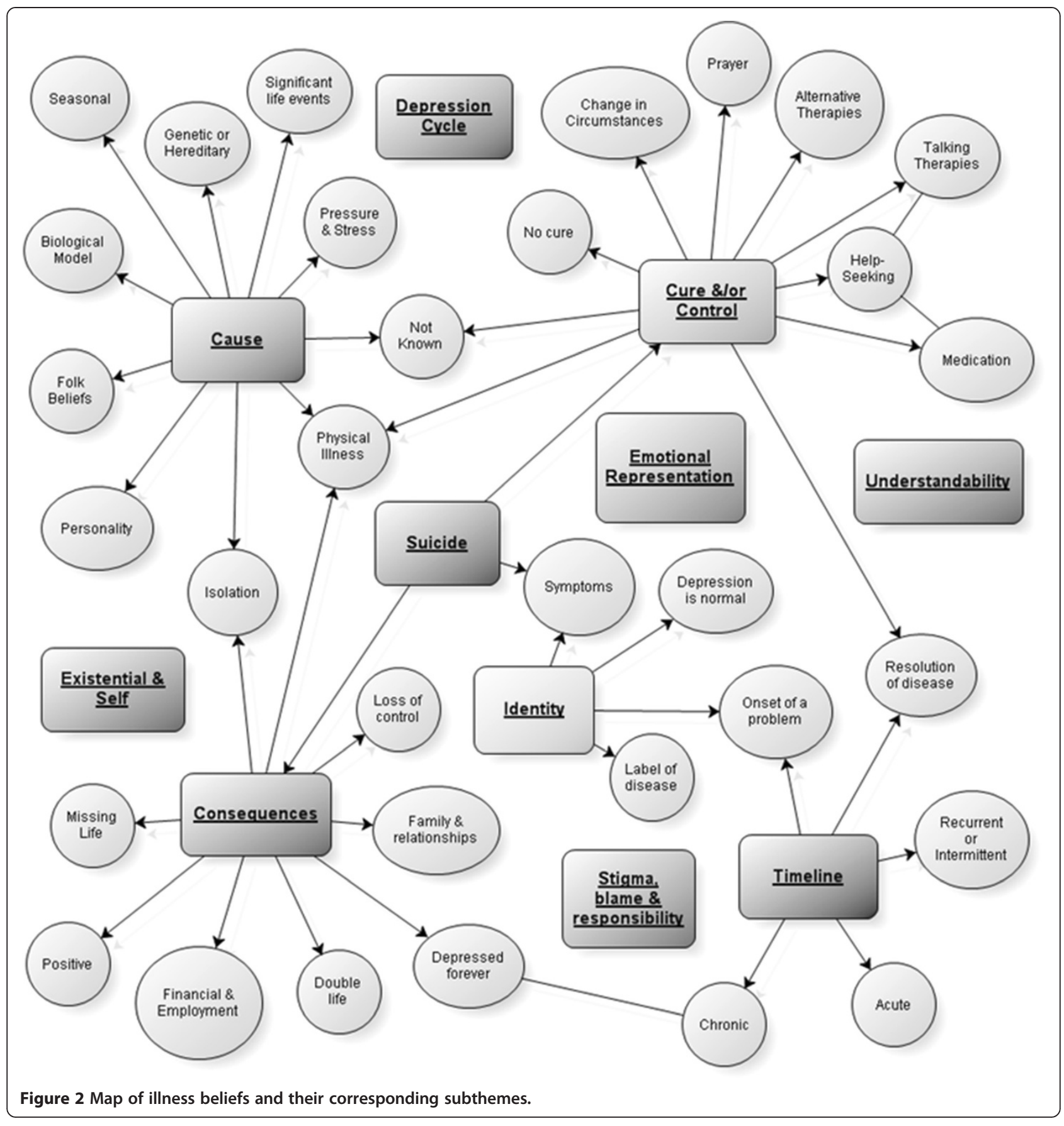

finding one that explained all of their experience" [55], leading to uncertainty about how to resolve problems.

Sometimes the account of depression contained several narrative episodes based on more than one storyline. [55]

\section{Depression cycle}

Depression was sometimes seen as a spiraling process, with episodes being both a consequence of previous depression and a cause of new onset of depression. These data could not be coded to the cause construct or the cyclical timeframe construct as one episode of depression was believed to be the cause of a subsequent episode of depression itself. Being depressed caused individuals to become more isolated and lonely, and made the sufferer further depressed. Having depression left the person with an internal weakness and predisposed to future depression, a cycle from which it was hard to break out. There are few other diseases where the disease itself can be seen in this way, and in this respect, this depression belief is unique. 
"Anxieties cause depression and depression causes selfdepreciation." [59]

\section{Existential and self}

This theme concerned the individual's sense of identity and differs from the identity of the disease theme. For some participants, even more than in physical diseases, depression is deeply interwoven in everyday life, in an existential understanding of the self and in a person's sense of social and individual identity. It defined the person as who they were in their entirety, not as a consequence of depression but more of a statement of their individuality. Having depression changed the way they viewed themselves and their personality. Depression gave them a new identity, and they joined a category of person in which they had not previously seen themselves. For many this was a distressing and unwelcome experience. On questioning, many participants strongly agreed that having depression affected the way they saw themselves as a person $[24,34]$.

"You know, I was a mental patient. That was my identity. . .depression is very private...It's no longer just my own pain. I am a mental patient. I am a depressive. I am a depressive [said slowly and with intensity]. This is my identity. I can't separate myself from that. When people know me they'll have to know about my psychiatric history, because that's who I am." [60]

"And when I came out I did feel quite odd because she gave me a prescription. I couldn't. I suddenly felt like I fell into a bracket of a type of people, emotionally in my head. Which is quite a strange feeling really because... I'm not like I thought I was and now I'm a bit different" [48].

\section{Suicidal thinking}

Suicide was rarely mentioned but when it was, it had an ambiguous status and did not fit within an obvious theme and was often a gender specific belief. Suicidal thinking was seen as symptom of depression, something people would never suffer with when well. For others, suicide was a consequence; the depression was so severe that suicide was an understandable response to suffering. It could also be seen as a control mechanism - a means of getting relief from their distress, and the most acceptable way of dealing with a problem. Suicide required a lot of self control and counteracted the image of being weak for having depression, particularly amongst males [54].

"In the beginning, you may not know what's happening to you... if it gets worse and you don't get help, people eventually hurt themselves with drugs or they can take their own life." [83]

"Men who kill themselves are doing what maybe a lot of men have been taught to do. Literally they are taking their lives in their own hands because that's what guys are taught to do. You know, to take care of $i t$, and they take care of it in a way that is absolutely what they believe to be the right thing to do." [54]

\section{Stigma, blame and responsibility}

Participants feared the outcome of others knowing about their condition. Depression was seen as poorly understood by the public, and misrepresented in the media, so that sufferers were to blame or responsible for their depression. This idea of blameworthiness was different from the situation when external factors were clearly contributing. For example, a severe economic depression in Finland was seen as being responsible for the increase in depression and was socially acceptable [55]. Participants were ashamed of being seen as not been able to cope - the stigma beliefs they had attached to people suffering from depression - and were now a part of themselves. Perceived stigma in itself had consequences, such as their judgment would no longer be trusted whilst they suffered from depression, leading to employment problems and the loss of friendships.

"When you have an operation you have friends who you can talk to. Last year, I had an accident and I received 45 get-well cards, but you go down with depression and nobody knocks on your door." [70] "The reason why it was hard to get psychiatric help was because of pride. I didn't want people to think, "How did he end up this bad?" I just don't want to be one of those crazy people, and it's basically admitting that I am not in charge of my own emotions." [76]

\section{Discussion}

\section{Summary of main findings}

Our most striking finding is the wide range of beliefs held by people experiencing symptoms when they are questioned, and importantly, although we started with a framework based upon how people think about illness, not all the beliefs we identified could be fitted into this structure. These themes have not been actively looked for in previous literature and therefore the number of studies contributing to each theme is small. They could not be fitted into the illness representations framework without losing some of the most interesting and potentially clinically significant beliefs about depression.

Our new themes support ideas from the health psychology literature. Sense of Coherence is said to assist individual coping with illness by facilitating understanding of the challenge of illness and by allowing the individual to integrate the illness experience with a sense of personal meaningfulness [89]. It is therefore closely related to our themes of understandability and existential and self. Sense of Coherence has been associated with good health and especially with good mental health [90]. 
Table 3 Implications derived from themes

\begin{tabular}{|c|c|}
\hline Theme & Implications \\
\hline Identity & $\begin{array}{l}\text { How patients think about depression and about being given a label or diagnosis for it may be important } \\
\text { in understanding why patients engage or do not engage in detection. }\end{array}$ \\
\hline Cause & $\begin{array}{l}\text { Mismatches in what patients and GPs believe causes depression may undermine the development } \\
\text { of shared treatment plans and undermine subsequent concordance. }\end{array}$ \\
\hline Cure \&/or Control & $\begin{array}{l}\text { Beliefs about the role and relevance of antidepressants or psychotherapy may affect whether patients wish } \\
\text { to have depressive symptoms detected. }\end{array}$ \\
\hline Timeline & $\begin{array}{l}\text { Patient beliefs about the course of their depression will affect detection. Those who expect quick resolution } \\
\text { may not think it to be appropriate to seek treatment. }\end{array}$ \\
\hline Consequences & $\begin{array}{l}\text { Negative views about the consequences of having depression may lead to hopelessness or defensiveness } \\
\text { in the face of attempts at standardised depression detection. }\end{array}$ \\
\hline Coherence & $\begin{array}{l}\text { Identifying how the patient thinks can be difficult in consultations, but it will be important to identify and } \\
\text { if possible moderate beliefs if they are not helpful to recovery. }\end{array}$ \\
\hline Depression Cycle & The cyclical beliefs leave patients feeling a sense of futility about long term approaches to intervention. \\
\hline Existential \& Self & $\begin{array}{l}\text { Discussing what depression means to how patients perceive themselves may increase acceptance by a patient } \\
\text { that depression can be a concern of clinicians. }\end{array}$ \\
\hline Role of suicide & $\begin{array}{l}\text { While suicidal acts are relatively rare, suicidal thoughts are relatively common. Exploring the latter is best with } \\
\text { an open mind towards their meaning for the patient. }\end{array}$ \\
\hline Stigma, blame \& responsibility & $\begin{array}{l}\text { Presenting screening as a normal and routine part of care may help reduce feelings of shame and } \\
\text { "give permission" to discuss depression. }\end{array}$ \\
\hline
\end{tabular}

There was no evidence that the selection criteria and depression status of the participants influenced beliefs. For example beliefs about whether depression symptoms are a normal part of life were endorsed in studies including participants who were recruited following selfdiagnosis of depression, a diagnosis in the medical records and those identified by screening or diagnostic interview. This suggests that beliefs about depression can be similar, regardless of whether a person is formally diagnosed.

\section{Comparison with existing literature}

We identified a greater diversity of beliefs than in previous review of the beliefs of people with current depression [16], perhaps because we actively looked for beliefs outside the framework of illness representations. That study also had a wider focus, including beliefs of the general public and those suffering from other problems such as anxiety [16].

\section{Strengths and limitations of the study}

Our review strengths include the comprehensive search strategy, the development of themes from methodologically robust studies, the systematic approach to synthesis and the integration of both qualitative and quantitative data. We used one increasingly common approach to integrating mixed data although others exist [19]. The sensitive search strategy employed meant that many non-relevant articles were found in the searches; however this has hopefully ensured that no relevant articles were missed.

Stage 1 identified only two studies with beliefs associated with a chronic physical illness [32,79]. So in stage 2 we included all depression beliefs. Beliefs associated with a physical illness may differ from those which are not, but the difference is likely to be on emphasis rather than in specific content. For example, symptoms of illness or its treatment may be seen as a cause of depression; or physical symptoms and depression may interact so that the consequences of their co-existence are felt more severely. Cause and consequence are existing themes in our framework, here given new content but not displaced by a new theme. Limiting the scope of this review to primary care may have meant that potentially relevant studies were missed but increased the relevance of this review to the current management of depression in primary care, such as case-finding for atrisk people. In the absence of established methodological consensus on whether or not to include quotations from original studies in a review of this type, we opted for inclusion to enhance illustration of the themes [91].

\section{Implications for further research and clinical practice}

As evidence accumulates [92] to show that chronic physical disease is a risk factor for depression, and that depression has a detrimental effect on morbidity and mortality, health professionals are likely to be encouraged to actively seek such at risk people. If we are unable to understand how patients think about depression and take into account their beliefs then the uptake and outcomes of depression screening are likely to be compromised, as well as patient concordance with any subsequent depression management. Particularly important are likely to be beliefs about the inappropriateness of having a quasi- 
diagnostic label, about the origins of depression in life problems and about medication being inappropriate. Equally important but often neglected is the evidence that not everybody thinks of depression as being illnesslike, such beliefs being incompatible in a more fundamental way with interventions based upon screen-treat approaches in healthcare. Our findings are relevant to patients with physical illnesses and we are undertaking two further studies to investigate beliefs about depression associated with a physical illness to pursue this. In Table 3 we summarize the clinical implications of our findings.

These considerations are important when there is a financial reward for administering a screening test without clear evidence of benefit, and when patients do not undergo an informed consent process which includes the risks and benefits of testing prior to the test being administered.

\section{Conclusions}

We need approaches to detection of depression in physical illness that are sensitive to the range of beliefs held by patients. Further research is needed to understand fully how people comprehend depression associated with a physical illness and how this influences help-seeking and engagement with health care services.

\section{Appendix 1 - search terms}

\section{A.1. Ovid MEDLINE (1950-present day)}

1) exp *Attitude to Health/

2) exp "Health Knowledge, Attitudes, Practice/

3) (illness adj2 (cognit* or schemat" or percept* or represent" or belie* or attitud* or behav" or reason")).tw.

4) (depress" adj2 (cognit* or schemat" or percept* or represent" or belie" or attitud* or behav" or reason")).tw.

5) exp "“Patient Acceptance of Health Care"/

6) exp "Models, Psychological/

7) (health belie* adj2 model*).tw.

8) (theor" adj2 plan" adj2 behav").tw.

9) (health" adj2 action" adj2 process").tw.

10) (social" adj2 cognit" adj2 model*).tw.

11) (protect" adj2 motiv" adj2 theor").tw.

12) (theor" adj2 reason" adj2 action").tw.

13) (common* adj2 sense").tw. 1676

14) (self" adj2 regulat").tw.

15) 1 or 2 or 3 or 4 or 5 or 6 or 7 or 8 or 9 or 10 or 11 or 12 or 13 or 14

16) exp *Depression/

17) exp "Depressive Disorder/

18) depress".tw.

19) 16 or 17 or 18

20) exp Family Practice/
21) exp Primary Health Care/

22) exp Physicians, Family/

23) ((general or family) adj practi\$).tw.

24) family physic\$.tw.

25) (primary adj2 care).tw.

26) (gp or gps).tw.

27) 20 or 21 or 22 or 23 or 24 or 25

28) 15 and 19 and 27

29) limit 28 to "all adult (19 plus years)"

\section{A.2. Ovid Embase (1980-present day)}

1) exp "attitude to health/

2) exp "health belief/

3) exp "Health Belief Model/

4) exp "patient attitude/

5) (illness adj2 (cognit" or schemat" or percept" or represent" or belie* or attitud* or behave* or reason*)).tw.

6) (depress* adj2 (cognit" or schemat* or percept* or represent" or belie" or attitud" or behave" or reason*)).tw.

7) exp psychological model/

8) (health belie* adj2 model*).tw.

9) (theor* adj2 plan* adj2 behav").tw.

10) (health" adj2 action" adj2 process").tw.

11) (social" adj2 cognit" adj2 model*).tw.

12) (protect" adj2 motivat" adj2 theor*).tw.

13) (theor" adj2 reason* adj2 action*).tw.

14) (common* adj2 sense*).tw.

15) (self" adj2 regulat*).tw.

16) 1 or 2 or 3 or 4 or 5 or 6 or 7 or 8 or 9 or 10 or 11 or 12 or 13 or 14 or 15

17) exp "depression/

18) depress".tw.

19) 17 or 18

20) exp general practice/

21) exp primary health care/

22) exp general practitioner/

23) ((general or family) adj pract*).tw.

24) family physic".tw.

25) (primary adj2 care).tw.

26) (gp or gps).tw.

27) 20 or 21 or 22 or 23 or 24 or 25 or 26

28) 16 and 19 and 27

\section{A.3. Ovid PsychINFO (1806-present day)}

1) exp *Client Attitudes/

2) exp *Consumer Attitudes/

3) exp "Health Attitudes/

4) exp "Health Knowledge/

5) exp "Mental Illness (Attitudes Toward)"/ 
6) (illness adj2 (cognit* or schemat" or percept" or represent* or belie* or attitud* or behave* or reason*)).tw.

7) (depress" adj2 (cognit* or schemat" or percept* or represent" or belie* or attitud" or behave* or reason")).tw.

8) (health belie* adj2 model*).tw.

9) (theor" adj2 plan" adj2 behav").tw.

10) (health" adj2 action" adj2 process").tw.

11) (social" adj2 cognit" adj2 model").tw.

12) (protect" adj 2 motiv* adj2 theor*).tw.

13) (theor* adj2 reason* adj2 action*).tw.

14) (common* adj2 sense*).tw.

15) (self* adj2 regulat*).tw.

16) 1 or 2 or 3 or 4 or 5 or 6 or 7 or 8 or 9 or 10 or 11 or 12 or 13 or 14 or 15

17) exp "major depression/

18) exp "“Depression (Emotion)"/

19) depress".tw.

20) 17 or 18 or 19

21) exp Primary Health Care/

22) exp General Practitioners/

23) exp Family Medicine/

24) exp Family Physicians/

25) ((general or family) adj practi*).tw.

26) family physic".tw.

27) (primary adj2 care).tw.

28) (gp or gps).tw.

29) 21 or 22 or 23 or 24 or 25 or 26 or 27

30) 16 and 20 and 29

31) limit 30 to adulthood $<18+$ years $>$

\section{A.4. EBSCO CINAHL (1982-present day)}

1) (MM "Attitude to Mental Illness")

2) MM "Patient Attitudes"

3) MM Attitude to health

4) TX (health belie* N2 model*) or TX (theor* N2 plan* N2 behav*) or TX (health* N2 action* N2 process") or TX (social* N2 cognit* N2 model*) or TX (protect* N2 motiv* N2 theor*) or TX (theor* N2 reason* N2 action") or TX (common* N2 sense*) OR TX (self* N2 regulat")

5) TX illness N2 cognit" or schemat" or percept" or represent" or belie* of attitud" or behav" or reason"

6) TX depress" N2 cognit" or schemat" or percept" or represent" or belie* or attitud" or behav" or reason"

7) 1 or 2 or 3 or 4 or 5 or 6

8) (MM "Depression+")

9) TX depress"

10) 8 or 9

11) (MH "Family Practice")

12) (MH "Primary Health Care")

13) (MH "Physicians, Family")
14) TX (general or family N2 practi*) or TX family physic* or TX primary N2 care or TX (gp or gps)

15) 11 or 12 or 13 or 14

16) 7 and 10 and 15

A.5. ISI web of science (including science citation index expanded, and conference proceedings citation index, 1898-present day)

1) Topic $=$ (attitude to mental illness)

2) Topic $=$ (patient attitudes)

3) Topic $=$ (attitude to health)

4) Topic $=$ (health knowledge)

5) Topic $=$ (patient acceptance of healthcare)

6) Topic $=(($ illness SAME (cognit" OR schemat" OR percept* OR represent* OR belie* OR attitud* OR behav" or reason*) $))$ OR Topic $=(($ depress" SAME (cognit* OR schemat" OR percept* OR represent* OR belie* OR attitud" OR behav" or reason*)))

7) Topic $=($ psychological models $)$ OR Topic $=(($ health belie* SAME model*) $)$ OR Topic $=(($ theor* SAME plan* SAME behave*)) OR TOPIC $=(($ health SAME action ${ }^{*}$ SAME process* $\left.)\right)$ OR Topic $=\left(\left(\left(\right.\right.\right.$ social ${ }^{*}$ SAME cognit* SAME model*)) OR Topic $=\left(\left(\right.\right.$ protect ${ }^{*}$ SAME motiv* SAME theor*) $\left.)\right)$ OR Topic $=(($ theor" SAME reason* SAME action*) $))$ OR Topic $=\left(\left(\right.\right.$ common* SAME sense $\left.\left.^{*}\right)\right)$ OR Topic $=(($ self* SAME regulat* $))$

8) 1 OR 2 OR 3 OR 4 OR 5 OR 6 OR 7

9) Topic $=($ depression $)$ OR Topic $=($ depress* $)$

10) Topic $=($ primary SAME care $)$ OR Topic $=($ Family SAME physic*) OR Topic $=($ gp OR gps) OR Topic $=(($ general OR family $)$ practice* $)$ OR Topic $=($ family SAME medic* $)$

11) 8 AND 9 AND 10

A.6. Cochrane library, Wiley InterScience, 2009 issue 4 (including the Cochrane database of systematic reviews, database of abstracts of reviews of effects, Cochrane central register of controlled trials, health technology assessment database, NHS economic evaluation database, and about the Cochrane collaboration)

1) Title - depress" AND (attitude* OR belie* OR percept* OR cognit* OR schemat* OR represent* OR behave* OR reason")

\section{BIOSIS (previews)}

1) exp Behavioral biology - Human behavior/

2) (illness adj2 (cognit" or schemat" or percept" or represent" or belie* or attitud" or behav" or reason*)).tw.

3) (depress* adj2 (cognit" or schemat* or percept* or represent" or belie* or attitud* or behav" or reason*)).tw. 
4) (health belie* adj2 model*).tw.

5) (theor" adj2 plan" adj2 behav").tw.

6) (health" adj2 action* adj2 process").tw.

7) (social adj2 cognit* adj2 theor*).tw.

8) (protect" adj2 motiv* adj2 theor*).tw.

9) (theor* adj2 reason* adj2 action*).tw.

10) (common* adj2 sense*).tw.

11) (self" adj2 regulat").tw.

12) 1 or 2 or 3 or 4 or 5 or 6 or 7 or 8 or 9 or 10 or 11

13) exp "Behavioral and Mental Disorders"/

14) exp Psychiatry/

15) depress".tw.

16) 13 or 14 or 15

17) ((general or family) adj practi*).tw.

18) family physic".tw.

19) (primary adj2 care).tw.

20) (gp or gps).tw.

21) 17 or 18 or 19 or 20

22) 12 and 16 and 21

23) limit 22 to human

24) limit 23 to adult

\section{A.7. The National Institute for Health Research Clinical Research Network Coordinating Centre (NIHR CRN CC)}

1) Topic - All

2) Title - depression

\section{A.8. The national research register archive}

1) Keywords - depressive disorder AND attitude-tohealth

\section{A.9. Www.ClinicalTrials.Gov}

1) Title - depression AND attitudes

\section{A.10. OpenSIGLE - grey literature}

1) Keyword = depression

\section{Competing interests}

All authors have completed the Unified Competing Interest form at www. icmje.org/coi_disclosure.pdf (available on request from the corresponding author) and declare: no support from any organisation for the submitted work; no financial relationships with any organisations that might have an interest in the submitted work in the previous three years, no other relationships or activities that could appear to have influenced the submitted work.

\section{Authors' contributions}

$\mathrm{AH}$ was responsible for the study conception and design, contributed to the interpretation of the data. RF, LG AH and KM commented on drafts of the manuscript. SA wrote the protocol and was responsible for data extraction, analysis and interpretation, drafting the manuscript and incorporating comments. RF and LG contributed to the interpretation of the data. KM acted as a second reviewer. SA will act as guarantor. All authors read and approved the final manuscript.
Funding

This project was not externally funded.

Received: 8 November 2011 Accepted: 12 April 2012

Published: 28 May 2012

\section{References}

1. National Institute of Clinical Excellence: Depression: Management of depression in primary and secondary care. In National Clinical Practice Guidelines. Volume 23. Edited by Natitional Institute for Clinical Excellence. London: National Institute for Clinical Excellence; 2004:362.

2. Goldney RD, Phillips PJ, Fisher LJ, Wilson DH: Diabetes, depression, and quality of life. Diabetes Care 2004, 27(5):1066-1070.

3. Rudisch B, Nemeroff CB: Epidemiology of comorbid coronary artery disease and depression. Biol Psychiatry 2003, 54(3):227-240.

4. Carney RM, Freedland KE, Miller GE, Jaffe AS: Depression as a risk factor for cardiac mortality and morbidity: a review of potential mechanisms. J Psychosom Res 2002, 53(4):897-902.

5. Whooley MA, Avins AL, Miranda J, Browner WS: Case-finding instruments for depression. Two questions are as good as many.[see comment]. J Gen Intern Med 1997, 12(7):439-445.

6. Department of Health: Talking therapies: a four-year plan of action. 2011.

7. Quality and outcomes framework - online GP practice results database., [http://www.qof.ic.nhs.uk/.

8. Subramanian DN, Hopayian K: An audit of the first year of screening for depression in patients with diabetes and ischaemic heart disease under the Quality and Outcomes Framework. Qual Prim Care 2008, 16(5):341-344.

9. Barley E, Murray J, Walters $P$, Tylee A: Managing depression in primary care: a meta-synthesis of qualitative and quantitative research from the UK to identify barriers and facilitators. BMC Fam Pract 2011, 12(1):47.

10. Dowrick C, Buchan I: Twelve month outcome of depression in general practice: does detection or disclosure make a difference? BMJ 1995, 311(7015):1274-1276.

11. Gilbody SM, House A, Sheldon T: Screening and case finding instruments for depression [Systematic Review]. Cochrane Database Syst Rev 2009, 3:3.

12. Pignone MP, Gaynes BN, Rushton JL, Burchell CM, Orleans CT, Mulrow CD, Lohr KN: Screening for depression in adults: a summary of the evidence for the U.S. Preventive Services Task Force. Ann Intern Med 2002, 136(10):765-776.

13. Kurtz S, Silverman J, Benson J, Draper J: Marrying content and process in clinical method teaching: enhancing the Calgary-Cambridge guides. Academic Med 2003, 78(8):802-809.

14. Lynch J, Kendrick T, Moore M, Johnston O, Smith PWF: Patients' beliefs about depression and how they relate to duration of antidepressant treatment. Use of a US measure in a UK primary care population. Primary Care Mental Health 2006, 4(3):207-217.

15. Johnston O, Kumar S, Kendall K, Peveler R, Gabbay J, Kendrick T: Qualitative study of depression management in primary care: GP and patient goals, and the value of listening. Br J Gen Pract 2007, 57(544):1E-14E.

16. Prins MA, Verhaak PFM, Bensing JM, van der Meer K: Health beliefs and perceived need for mental health care of anxiety and depression - The patients' perspective explored. Clin Psychol Rev 2008, 28(6):1038-1058.

17. Liberati A, Altman DG, Tetzlaff J, Mulrow C, Gotzsche PC, loannidis JPA, Clarke M, Devereaux PJ, Kleijnen J, Moher D: The PRISMA statement for reporting systematic reviews and meta-analyses of studies that evaluate healthcare interventions: explanation and elaboration. Br Med J 2009, 339:37.

18. NHS Centre for Reviews and Dissemination: Systematic Reviews: CRD's guidance for undertaking reviews in health care. In. 2nd editionEdited by NHS Centre for Reviews and Dissemination. York: University of York; 2001:292.

19. Popay J, Roberts H, Sowden A, Petticrew M, Arai L, Rodgers M, Britten N, Roen K, Steven D: Guidance on the Conduct of Narrative Synthesis in Systematic Reviews. 1st edition. ESRC Methods: Lancaster; 2006.

20. Cameron LD, Leventhal H: The Self-Regulation of Health and IIIness Behaviour: Routledge; 2003.

21. Hampson SE: Illness Representations and the Self-Management of Diabetes. In Perceptions of Health and IIIness. Edited by Petrie KJ, Weinman J. Amsterdam: Harwood Academic Publishers; 1997:323-348. 
22. Petrie KJ, Weinman JA: Perceptions of Health and IIIness. 1st edition. Amsterdam: Harwood Academic Publishers; 1997.

23. Lobban F, Barrowclough C, Jones S: A review of the role of illness models in severe mental illness. Clin Psychol Rev 2003, 23(2):171-196.

24. Brown C, Dunbar-Jacob J, Palenchar DR, Kelleher KJ, Bruehlman RD, Sereika S, Thase ME: Primary care patients' personal illness models for depression: a preliminary investigation. Fam Pract 2001, 18(3):314-320.

25. Addis ME, Truax P, Jacobson NS: Why do people think they are depressed? The reasons for depression questionnaire. Psychotherapy 1995, 32(3):476-483.

26. Addis ME, JN S: Reasons for depression and the process and outcome of cognitive-behavioral psychotherapies. J Consult Clin Psychol 1996, 64:1417-1424.

27. Allen RL, Walker Z, Shergill SS, D'Ath P, Katona CLE: Attitudes to depression in hospital inpatients: a comparison between older and younger subjects. Aging Ment Health 1998, 2(1):36-39.

28. Al-Saffar N, Deshmukh A, Eid S, Carter P: Health beliefs and drug compliance of depressed patients in Kuwait. J Social Administrative Pharm 2003, 20(4):142-150.

29. Backenstrass M, Joest $K$, Rosemann T, Szecsenyi J: The care of patients with subthreshold depression in primary care: is it all that bad? A qualitative study on the views of general practitioners and patients. BMC Health Serv Res 2007, 7:190.

30. Badger F, Nolan P: Use of self-chosen therapies by depressed people in primary care. J Clin Nurs 2007, 16(7):1343-1352.

31. Bann CM, Parker CB, Bradwejn J, Davidson JR, Vitiello B, Gadde KM: Assessing patient beliefs in a clinical trial of Hypericum perforatum in major depression. In Depress Anxiety.: ; 2004:122-144.

32. Bogner HR, Dahlberg B, de Vries HF, Cahill E, Barg FK: Older patients' views on the relationship between depression and heart disease. Fam Med 2008, 40(9):652-657.

33. Brown C, Battista DR, Bruehlman R, Sereika SS, Thase ME, Dunbar-Jacob J: Beliefs about antidepressant medications in primary care patients: relationship to self-reported adherence. Med Care 2005, 43(12):1203-1207.

34. Brown C, Battista DR, Sereika SM, Bruehlman RD, Dunbar-Jacob J, Thase ME: Primary care patients' personal illness models for depression: relationship to coping behavior and functional disability. Gen Hospital Psychiatry 2007, 29(6):492-500.

35. Burroughs $H$, Lovell K, Morley M, Baldwin R, Burns A, Chew-Graham C: 'Justifiable depression': how primary care professionals and patients view late-life depression? A qualitative study. Fam Pract 2006, 23(3):369-377.

36. Cabassa LJ, Lagomasino IT, Dwight-Johnson M, Hansen MC, Xie B: Measuring Latinos' perceptions of depression: a confirmatory factor analysis of the IIIness Perception Questionnaire. Cultur Divers Ethni Minor Psychol 2008, 14(4):377-384.

37. Cape J, McCulloch Y: Patients' reasons for not presenting emotional problems in general practice consultations. Br J Gen Pract 1999, 49 (448):875-879.

38. Chakraborty K, Avasthi A, Kumar S, Grover S: Attitudes and beliefs of patients of first episode depression towards antidepressants and their adherence to treatment. Social Psychiatry and Psychiatric Epidemiol 2009, 44(6):482-488

39. Cooper LA, Brown C, Vu HT, Ford DE, Powe NR: How important is intrinsic spirituality in depression care? A comparison of white and AfricanAmerican primary care patients. In 15th Annual Meeting of the Associationfor-Health-Services-Research: Jun 21-23 1998. Washington, D.C: Blackwell Science Inc; 1998:634-638.

40. Cooper LA, Brown C, Vu HT, Palenchar DR, Gonzales JJ, Ford DE, Powe NR: Primary care patients' opinions regarding the importance of various aspects of care for depression. Gen Hosp Psychiatry 2000, 22(3):163-173.

41. Cooper LA, Gonzales JJ, Gallo JJ, Rost KM, Meredith LS, Rubenstein LV, Wang N, Ford DE: The acceptability of treatment for depression among African-American, Hispanic, and white primary care patients. Med Care 2003, 41(4):479-489.

42. Cooper-Patrick L, Powe NR, Jenckes MW, Gonzales JJ, Levine DM, Ford DE: Identification of patient attitudes and preferences regarding treatment of depression. J Gen Intern Med 1997, 12(7):431-438.

43. Cornford CS, Hill A, Reilly J: How patients with depressive symptoms view their condition: a qualitative study. Fam Pract 2007, 24(4):358-364

44. Danielsson U, Bengs C, Lehti A, Hammarstrom A, Johansson EE: Struck by lightning or slowly suffocating - gendered trajectories into depression. BMC Fam Pract 2009, 10:56
45. Dejman M, Ekbad S, Forouzan AS, Baradaran-Eftekhari M, Malekafzali H: Explanatory model of help-seeking and coping mechanisms among depressed women in three ethnic groups of Fars, Kurdish, and Turkish in Iran. Arch Iran Med 2008, 11(4):397-406.

46. Edlund MJ, Fortney JC, Reaves CM, Pyne JM, Mittal D: Beliefs about depression and depression treatment among depressed veterans. Med Care 2008, 46(6):581-589.

47. Fortune G, Barrowclough C, Lobban F: Illness representations in depression. Br J Clin Psychol 2004, 43:347-364

48. Garfield SF, Smith FJ, Francis S: The paradoxical role of antidepressant medication - returning to normal functioning while losing the sense of being normal. J Ment Heal 2003, 12(5):521-535.

49. Gask L, Rogers A, Oliver D, May C, Roland M: Qualitative study of patients' perceptions of the quality of care for depression in general practice. $\mathrm{Br} J$ Gen Pract 2003, 53(489):278-283.

50. Givens UL, Datto CJ, Ruckdeschel K, Knott K, Zubritsky C, Oslin DW, Nyshadham S, Vanguri P, Barg FK: Older patients' aversion to antidepressants. A qualitative study. J Gen Intern Med 2006, 21(2):146-151.

51. Givens JL, Houston TK, Van Voorhees BW, Ford DE, Cooper LA: Ethnicity and preferences for depression treatment. Gen Hosp Psychiatry 2007, 29(3):182-191.

52. Green G, Bradby H, Chan A, Lee M, Eldridge K: Is the English National Health Service meeting the needs of mentally distressed Chinese women? J Health Serv Res Policy 2002, 7(4):216-221.

53. Grime J, Pollock K: Patients' ambivalence about taking antidepressants: a qualitative study. Pharma J 2003, 271(7270):516-519.

54. Heifner C: The male experience of depression. Perspect Psychiatric Care 1997, 33(2):10-18.

55. Kangas I: Making sense of depression: perceptions of melancholia in lay narratives. Health 2001, 5(1):76-92.

56. Karasz A, Sacajiu G, Garcia N: Conceptual models of psychological distress among low-income patients in an inner-city primary care clinic. J Gen Intern Med 2003, 18(6):475-477.

57. Karasz A, Watkins L: Conceptual models of treatment in depressed hispanic patients. Ann Fam Med 2006, 4(6):527-533.

58. Karasz A: The development of valid subtypes for depression in primary care settings: a preliminary study using an explanatory model approach. J Nerv Ment Dis 2008, 196(4):289-296.

59. Karasz A, Garcia N, Ferri L: Conceptual models of depression in primary care patients a comparative study. J Cross-Cultural Psychology 2009, 40(6):1041-1059.

60. Karp DA: Living with depression: illness and identity turning points. Qual Health Res 1994, 4(1):6-30.

61. Kelly MA, Sereika SM, Battista DR, Brown C: The relationship between beliefs about depression and coping strategies: Gender differences. $\mathrm{Br} J$ Clin Psychol 2007, 46(3):315-332.

62. Kirk LB: Attitudes toward depression and its treatment in disadvantaged, depressed women. In Dissertation Abstracts International.: ; 2001:4409.

63. Kuyken W, Brewin CR, Power MJ, Furnham A: Causal beliefs about depression in depressed-patients, clinical psychologists and lay persons. Br J Med Psychol 1992, 65:257-268.

64. Lewis SE: A search for meaning: making sense of depression. J Ment Heal 1995, 4(4):369-382.

65. Leykin Y, DeRubeis RJ, Shelton RC, Amsterdam JD: Changes in patients' beliefs about the causes of their depression following successful treatment. In Cognitive Therapy and Research.: ; 2007:437-449.

66. Lowe B, Schulz U, Grafe K, Wilke S: Medical patients' attitudes toward emotional problems and their treatment. What do they really want? J Gen Intern Med 2006, 21(1):39-45.

67. Manber R, Chambers AS, Hitt SK, McGahuey C, Delgado P, Allen JJB: Patients' perception of their depressive illness. J Psychiatr Res 2003, 37(4):335-343

68. Martin D, Quirino J, Mari J: Depression among women living in the outskirts of Sao Paulo, Southeastern Brasil. Rev Saude Publica 2007, 41(4):591-597

69. Maxwell M: Women's and doctors' accounts of their experiences of depression in primary care: the influence of social and moral reasoning on patients' and doctors' decisions. Chronic Illness 2005, 1(1):61-71.

70. Nolan P, Badger F: Aspects of the relationship between doctors and depressed patients that enhance satisfaction with primary care. J Psychiatr Ment Health Nurs 2005, 12(2):146-153. 
71. Okello ES, Neema S: Explanatory models and help-seeking behavior: Pathways to psychiatric care among patients admitted for depression in Mulago hospital, Kampala, Uganda. Qual Health Res 2007, 17(1):14-25.

72. Pang KYC: Symptoms of depression in elderly Korean immigrants: Narration and the healing process. Cult Med Psychiatr 1998, 22(1):93-122.

73. Rogers A, May C, Oliver D: Experiencing depression, experiencing the depressed: the separate worlds of patients and doctors. J Ment Heal 2001, 10(3):317-333.

74. Sarkisian CA, Lee-Henderson MH, Mangione CM: Do depressed older adults who attribute depression to "old age" believe it is important to seek care? J Gen Intern Med 2003, 18(12):1001-1005.

75. Scattolon Y, Stoppard JM: "Getting on with life": Women's experiences and ways of coping with depression. Can Psychol-Psychol Can 1999, 40(2):205-219.

76. Shin JK: Help-seeking behaviors by Korean immigrants for depression. Issues Ment Health Nurs 2002, 23(5):461-476.

77. Srinivasan J, Cohen NL, Parikh SV: Patient attitudes regarding causes of depression: implications for psychoeducation. Can J Psychiatry 2003, 48(7):493-495.

78. Stecker T, Alvidrez J: Patient decision-making regarding entry into psychotherapy to treat depression. Issues Ment Health Nurs 2007, 28 (7):811-820.

79. Ugarriza DN: Elderly women's explanation of depression. J Gerontol Nurs 2002, 28(5):22-29. quiz 54-25.

80. Van Voorhees BW, Fogel J, Houston TK, Cooper LA, Wang NY, Ford DE: Beliefs and attitudes associated with the intention to not accept the diagnosis of depression among young adults. Ann Fam Med 2005, 3(1):38-46.

81. Van Voorhees BW, Fogel J, Houston TK, Cooper LA, Wang NY, Ford DE: Attitudes and illness factors associated with low perceived need for depression treatment among young adults. Social Psychiatry and Psychiatric Epidemiol 2006, 41(9):746-754.

82. Wagner PJ, Jester D, LeClair B, Taylor AT, Woodward L, Lambert J: Taking the edge off - Why patients choose St. John's Wort. J Fam Pract 1999, 48(8):615-619.

83. Waite R, Killian P: Perspectives about depression: explanatory models among African-American women. Arch Psychiatr Nurs 2009, 23(4):323-333

84. Williams B, Healy D: Perceptions of illness causation among new referrals to a community mental health team: "explanatory model" or "exploratory map"? Soc Sci Med 2001, 53(4):465-476.

85. Wittink MN, Dahlberg B, Biruk C, Barg FK: How older adults combine medical and experiential notions of depression. Qual Health Res 2008, 18(9):1174-1183

86. Wittkampf $K$, van Zwieten $M$, Smits F, Schene A, Huyser J, van Weert $H$ : Patients' view on screening for depression in general practice. Fam Pract 2008, 25(6):438-444.

87. Yeung A, Chang D, Gresham RL Jr, Nierenberg AA, Fava M: Illness beliefs of depressed Chinese American Patients in primary care. $J$ Nerv Ment Dis 2004, 192(4):324-327.

88. Martin D, de Jesus Mari J, Quirino J: Views on depression among patients diagnosed as depressed in a poor town on the outskirts of Sao Paulo, Brazil. Transcult Psychiatry 2007, 44(4):637-658.

89. Antonovsky A: Health, Stress, and Coping.: Jossey-Bass; 1979

90. Lindstrom B, Eriksson M: Contextualizing salutogenesis and Antonovsky in public health development. Heal Promot Int 2006, 21(3):238-244.

91. : Integrative approaches to qualitative and quantitative evidence., [http:// www.nice.org.uk/page.aspx?o=508055].

92. National Institute of Clinical Excellence: Depression in adults with a chronic physical health problem: treatment and management. In NICE. volume CG 91.: NICE; 2009.

doi:10.1186/1471-2296-13-41

Cite this article as: Alderson et al: How patients understand depression associated with chronic physical disease - a systematic review. BMC Family Practice 2012 13:41.

\section{Submit your next manuscript to BioMed Central and take full advantage of:}

- Convenient online submission

- Thorough peer review

- No space constraints or color figure charges

- Immediate publication on acceptance

- Inclusion in PubMed, CAS, Scopus and Google Scholar

- Research which is freely available for redistribution

Submit your manuscript at www.biomedcentral.com/submit
() Biomed Central 